\title{
A comprehensive gene mutation analysis of liquid biopsy samples from patients with metastatic colorectal cancer to the ovary: A case report
}

\author{
NAOYUKI IWAHASHI ${ }^{1}$, KAZUKO SAKAI $^{2}$, TOMOKO NOGUCHI ${ }^{1}$, TAMAKI YAHATA ${ }^{1}$, \\ SAORI TOUJIMA $^{1}$, KAZUTO NISHIO $^{2}$ and KAZUHIKO INO ${ }^{1}$ \\ ${ }^{1}$ Department of Obstetrics and Gynecology, Wakayama Medical University, Wakayama 641-0012; \\ ${ }^{2}$ Department of Genome Biology, Kindai University Faculty of Medicine, Osaka 589-8511, Japan
}

Received May 9, 2018; Accepted September 17, 2018

DOI: $10.3892 / \mathrm{ol} .2018 .9467$

\begin{abstract}
Liquid biopsies of circulating tumor DNA (ctDNA) can detect molecular alterations, including tumor-specific mutations, and have recently been used as a non-invasive diagnostic, prognostic, and predictive tool. However, this technique is not commonly used in the gynecological field. Gene mutation profiling of liquid biopsy samples was performed using CAncer Personalized Profiling by deep Sequencing (CAPP-Seq), a novel next-generation sequencing-based approach to ultrasensitive ctDNA detection, in order to make it possible to molecularly diagnose metastatic colorectal cancer to the ovary. Liquid biopsy (plasma) samples and formalin-fixed paraffin-embedded tumor samples were obtained from two patients with ovarian tumors, who had a history of surgery for colorectal cancer, and comprehensive gene mutation profiling was conducted using CAPP-Seq. In patient 1 , mutations were identified in the same three regions in both the ovarian tumor and preoperative plasma sample (in the KRAS G13D, APC E1306*, and TP53 H193Y genes). In patient 2 , mutation was identified in the same one region in all the primary colorectal tumor, the ovarian tumor, and preoperative plasma sample (in APC R $216^{*}$ gene). These mutations are well-known genetic signatures of colorectal cancer, suggesting that the ovarian tumor was metastatic. The gene mutation patterns of colorectal cancer were examined by subjecting liquid biopsy samples from patients with suspected metastatic ovarian tumors to CAPP-Seq. Gene mutation profiling of liquid biopsy samples can contribute to the preoperative differential
\end{abstract}

Correspondence to: Dr Naoyuki Iwahashi, Department of Obstetrics and Gynecology, Wakayama Medical University, 811-1 Kimiidera, Wakayama 641-0012, Japan

Email: naoyuki@wakayama med.ac.jp

Key words: metastatic ovarian cancer, circulating tumor DNA, liquid biopsy, comprehensive gene mutation analysis, cancer personalized profiling by deep sequencing diagnosis of metastatic ovarian cancer and its subsequent personalized treatment.

\section{Introduction}

The incidence of metastatic ovarian cancer varies greatly across different studies (it was reported to occur in 2.3 to $23.7 \%$ of cases of malignant ovarian cancer). Such tumors mainly arise from the colon (10.9 to $33.2 \%$ ), breast (1.8 to 33.3\%), or stomach (4.5 to $30.4 \%$ ) (1). Metastatic ovarian cancer occurs in 5 to $9.7 \%$ of women with colorectal cancer, who exhibit a median survival period of only 19 to 27 months after detection $(2,3)$. Surgical treatment has been recommended in several retrospective studies because it results in a better prognosis (4-6), and chemotherapy exhibits poor sensitivity, although recurrence and distant metastasis often occur during the follow-up period. Furthermore, the preoperative clinical diagnosis of metastatic ovarian cancer is difficult, and pathological examinations of surgical samples are required to definitively differentiate it from primary ovarian cancer. In previous studies of its utility for distinguishing primary ovarian cancer from metastatic ovarian cancer, imaging analysis was shown to only produce non-specific findings $(7,8)$.

Despite recent progress in colorectal cancer treatment, including the development of molecular-targeting therapy, there are little available data about the best way to treat patients affected by metastatic colorectal cancer to the ovary. Furthermore, there is insufficient information about the clinicopathological and genomic predictors of the outcomes of such patients, and the optimal clinical management strategy remains unclear $(9,10)$. Ganesh et al $(9)$, examined the genetic aberrations found in metastatic colorectal cancer to the ovary, including primary colorectal cancer, ovarian metastasis, and extra-ovarian metastasis. The frequencies of somatic mutations in oncogenes and tumor suppressor genes demonstrated a high concordance rate between matched primary and metastatic (from sites other than the ovary) colorectal tumors. Increased frequencies of mutations were seen in the Kirsten rat sarcoma viral oncogene homolog $(K R A S)$, Smad family member 4 (SMAD4), and neurotrophic receptor tyrosine kinase 1 (NTRK1) genes. 
Recent studies have shown that genomic alterations in solid cancers can be characterized by massive parallel sequencing of the circulating cell-free tumor DNA released from cancer cells in plasma, which is known as circulating tumor DNA (ctDNA). This technique is effectively a non-invasive 'liquid biopsy' examination $(11,12)$. Liquid biopsy examinations of ctDNA can be used to detect molecular alterations, including tumor-specific mutations, and have recently been used as a diagnostic, prognostic, and predictive tool, especially in colorectal cancer (to detect $R A S$ mutations) and lung cancer (to detect epidermal growth factor receptor [EGFR] mutations) (13-15). However, to the best of our knowledge, there have not been any reports about the use of liquid biopsy examinations in patients with metastatic colorectal cancer to the ovary. In this study, we subjected plasma ctDNA samples (liquid biopsy samples) to multiple gene mutation analysis using CAncer Personalized Profiling by deep Sequencing (CAPP-Seq), a novel next-generation sequencing-based approach to ultrasensitive ctDNA detection $(16,17)$, in patients with ovarian metastases from colorectal cancer. Then, we compared the findings with the results obtained for primary and metastatic tumor samples.

\section{Materials and methods}

Patients and samples. Two patients whose metastatic ovarian tumors had been surgically resected at Wakayama Medical University Hospital between May 2017 and June 2017 were included in this study. The tumor staging was carried out according to the TNM classification. The present study was approved by the ethics committee of Wakayama Medical University Faculty of Medicine (authorization no: 2025) and Kindai University Faculty of Medicine (authorization no: 29-066). All of the patients in this study provided written informed consent for the use of their plasma and tissue samples.

Tumor DNA extraction. Formalin-fixed paraffin-embedded (FFPE) specimens were subjected to a histological review, and only those containing sufficient tumor cells (at least $75 \%$ tumor cells), as determined by hematoxylin and eosin staining, were used for DNA extraction. The collected DNA was purified with the use of an AllPrep DNA/RNA FFPE kit (Qiagen, Inc., Valencia, CA, USA), according to the manufacturer's instructions. The quality and quantity of the DNA were verified using the NanoDrop 2000 device (Thermo Fisher Scientific, Inc., Wilmington, DE, USA) and PicoGreen dsDNA assay kit (Thermo Fisher Scientific, Inc.). The extracted DNA was stored at $-80^{\circ} \mathrm{C}$ until the analysis.

Tumor DNA sequencing. We used a 40 ng of DNA for the QIAseq Human Comprehensive Cancer Panel (275 genes; Qiagen, Inc.). Library preparation was performed according to the manufacturer's instructions. The purified libraries were pooled and then sequenced with a NextSeq 500 instrument (Illumina, Inc., San Diego, CA, USA). Reads were aligned with the hg19 human reference genome, and variant detection was performed according to the manufacturer's pipeline (18). Germline mutations were excluded with the use of the Human Genetic Variation Database (http://www.genome.med.kyoto-u.ac.jp/SnpDB) and the Exome Aggregation Consortium database (19).
ctDNA extraction. Samples $(8.5 \mathrm{ml})$ of peripheral blood were collected from the patients in cell-free DNA collection tubes (Roche Diagnostics, Indianapolis, IN, USA). Plasma ctDNA was purified using an AVENIO cfDNA isolation kit (Roche Diagnostics), according to the manufacturer's instructions. The quality and quantity of the DNA were verified using the NanoDrop 2000 device (Thermo Fisher Scientific, Inc.) and PicoGreen dsDNA assay kit (Thermo Fisher Scientific, Inc.). The extracted ctDNA was stored at $-80^{\circ} \mathrm{C}$ until the analysis.

ctDNA sequencing. We used a maximum of $50 \mathrm{ng}$ of DNA for the CAPP-Seq ctDNA analyses using the AVENIO ctDNA surveillance kit (197 genes; Roche Diagnostics), according to the manufacturer's instructions. The purified libraries were pooled and sequenced on an Illumina NextSeq 500 (Illumina, Inc.) using the 300-cycle high output kit. Variants were called with the AVENIO ctDNA analysis software (Roche Diagnostics), which includes bioinformatics methods from CAPP-Seq (16) and integrated digital error suppression (17). Germline mutations were excluded with the use of the Human Genetic Variation Database (http://www.genome.med.kyoto-u. ac.jp/SnpDB) and the Exome Aggregation Consortium database (19).

\section{Results}

Patient 1. A 61-year-old female was referred to our institution with suspected ovarian cancer. She had been diagnosed with sigmoid colon cancer 2 years ago, which was completely surgically resected in other institution. The tumor had a moderately differentiated histology, and pathologically, it extended into the subserosa (pT3). There were no lymph node or distant metastases ( $\mathrm{pN} 0, \mathrm{M} 0)$. The patient received adjuvant chemotherapy (8 courses of capecitabine). Two years later, a tumor appeared in the right ovary. Magnetic resonance imaging (MRI) revealed a solid 4.3-cm mass with an irregular surface, and positron emission tomography-computed tomography (PET-CT) detected high radiotracer uptake by the tumor [maximum standardized uptake value (SUVmax): 10.66]. The patient's levels of carcinoembryonic antigen (CEA) and cancer antigen (CA) 19-9 were elevated (to $8.8 \mathrm{ng} / \mathrm{ml}$ and $58.4 \mathrm{U} / \mathrm{ml}$, respectively), whereas her CA125 level was normal. The patient underwent a total abdominal hysterectomy and bilateral salpingo-oophorectomy, and the pathological diagnosis was metastatic ovarian cancer. We obtained an FFPE sample from the metastatic ovarian tumor $(\mathrm{O}-1)$ and a preoperative plasma ctDNA sample (L-1). The patient's clinical course and clinicopathological features are summarized in Fig. 1A. Targeted re-sequencing was performed using the QIAseq Human Comprehensive Cancer Panel for O-1 and the AVENIO ctDNA surveillance kit for L-1. Intronic changes and exonic SNP listed in the HGVD database were excluded from our analysis, as described in Materials and Methods. Non-synonymous somatic point mutations that resulted in changes in protein amino acid sequences are summarized in Table I. In O-1, mutations were identified in three regions, the KRAS G13D, adenomatous polyposis coli (APC) E1306*, and tumor protein p53 (TP53) H193Y genes. Sequencing of the plasma ctDNA detected three mutations and one copy number variation; i.e., KRAS G13D, APC E1306*, and TP53 H193Y mutations and 
A

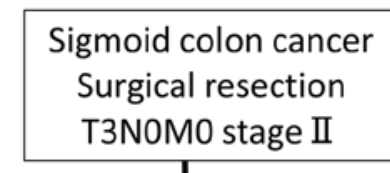

Capecitabine

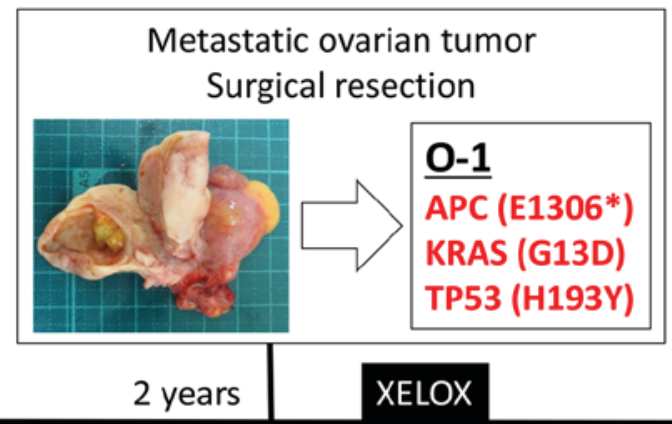

Tumor

Liquid

B
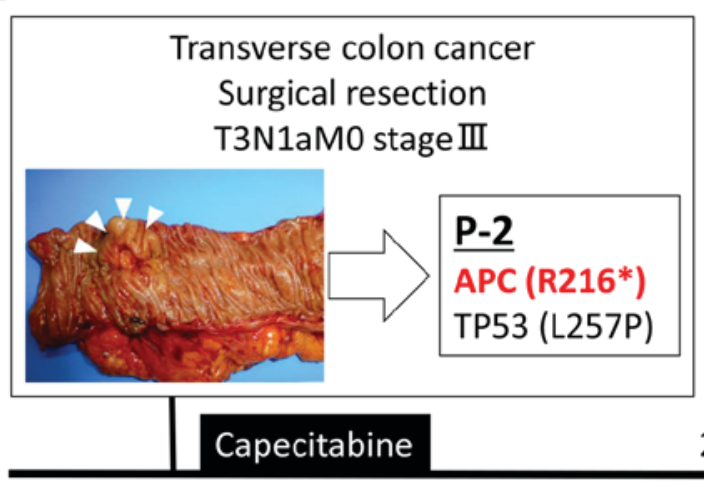
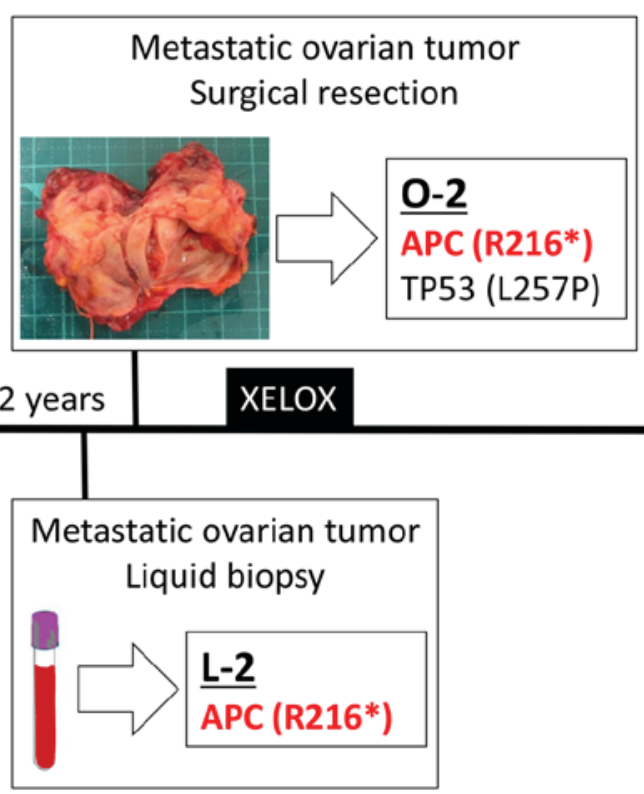

Tumor

Liquid

Figure. 1. Treatment timelines for the two patients. (A) Patient 1 (a 61-year-old female) had previously been diagnosed with sigmoid colon cancer, which was surgically resected. The patient received adjuvant chemotherapy ( 8 courses of capecitabine). Two years later, ovarian metastasis was detected, and the patient underwent surgical resection (O-1), followed by adjuvant chemotherapy (8 courses of XELOX). A liquid sample (plasma) was collected preoperatively (L-1). (B) Patient 2 (a 66-year-old female) had previously been diagnosed with primary transverse colon cancer, which was surgically resected (P-2). The patient received adjuvant chemotherapy (8 courses of capecitabine). Two years later, ovarian metastasis was detected, and the patient underwent surgical resection (O-2), followed by adjuvant chemotherapy (8 courses of XELOX). A liquid sample (plasma) was collected preoperatively (L-2).

MET gene amplification. A comparison of the genetic profiles of O-1 and L-1 revealed that the KRAS G13D, APC E1306*, and TP53 H193Y gene mutations, which are well-known genetic signatures of colorectal cancer were present in both O-1 and L-1. These results suggest that liquid biopsy-based gene mutation profiling could facilitate the preoperative diagnosis of metastatic colorectal cancer to the ovary. The patient received postoperative adjuvant chemotherapy ( 8 courses of capecitabine and oxaliplatin; XELOX).
Patient 2. A 66-year-old female was referred to our institution with suspected ovarian cancer. She had been diagnosed with transverse colon cancer 2 years earlier, which was completely surgically resected. The tumor had a well-differentiated histology, and pathologically, it extended into the subserosa (pT3). There was a single lymph node metastasis, but no distant metastasis (pN1a, M0). The patient was treated with adjuvant chemotherapy ( 8 courses of capecitabine). Two years later, a tumor appeared in the right ovary. MRI revealed a 
Table I. Non-synonymous mutations in Patient 1.

\begin{tabular}{|c|c|c|c|c|c|c|}
\hline \multirow[b]{3}{*}{ Chromosome } & \multirow[b]{3}{*}{ Position } & \multirow[b]{3}{*}{ Gene } & \multirow[b]{3}{*}{ Variant effect } & \multirow[b]{3}{*}{ Amino acid change } & \multicolumn{2}{|c|}{ Variant frequency $(\%)$} \\
\hline & & & & & Liquid & Ovary \\
\hline & & & & & L-1 & O-1 \\
\hline Chr5 & 112839510 & APC & Nonsense & E1306* & 11.85 & 41.2 \\
\hline Chr12 & 25245347 & KRAS & Missense & G13D & 7.58 & 26.0 \\
\hline Chr17 & 7674954 & TP53 & Missense & H193Y & 9.48 & 41.0 \\
\hline
\end{tabular}

Chr, chromosome.

Table II. Non-synonymous mutations in Patient 2.

\begin{tabular}{|c|c|c|c|c|c|c|c|}
\hline \multirow[b]{3}{*}{ Chromosome } & \multirow[b]{3}{*}{ Position } & \multirow[b]{3}{*}{ Gene } & \multirow[b]{3}{*}{ Variant effect } & \multirow[b]{3}{*}{ Amino acid change } & \multicolumn{3}{|c|}{ Variant frequency $(\%)$} \\
\hline & & & & & Colon & Liquid & Ovary \\
\hline & & & & & $\mathrm{P}-2$ & L-2 & $\mathrm{O}-2$ \\
\hline Chr5 & 112792446 & APC & Nonsense & $\mathrm{R} 216^{*}$ & 17.2 & 0.11 & 20.3 \\
\hline Chr17 & 7577511 & TP53 & Missense & L257P & 23.6 & 0 & 28.5 \\
\hline
\end{tabular}

Chr, chromosome.

multilocular, partially solid, 5.0-cm mass with an irregular surface, and PET-CT detected high radiotracer uptake by the tumor (SUVmax: 6.39). The patient's CEA level was elevated (11.1 ng/ml), while her CA19-9 and CA125 levels were normal. She underwent a total abdominal hysterectomy and bilateral salpingo-oophorectomy, and the pathological diagnosis was metastatic ovarian cancer. We obtained an FFPE sample of the primary transverse colon cancer (P-2), an FFPE sample of the metastatic ovarian tumor (O-2), and a plasma ctDNA sample before the second operation (L-2). The patient's clinical course and clinicopathological features are summarized in Fig. 1B. Targeted re-sequencing was performed using the QIAseq Human Comprehensive Cancer Panel for the FFPE samples and the AVENIO ctDNA surveillance kit for the plasma ctDNA. The intronic changes and exonic SNP listed in the HGVD database were excluded from our analysis, as described in Materials and Methods. The non-synonymous somatic point mutations that resulted in changes in protein amino acid sequences are summarized in Table II. Mutations were identified in the same region; i.e., $A P C \mathrm{R} 216^{*}$ and TP53 L257P, in both the primary (P-2) and metastatic (O-2) tumors. Sequencing of the plasma ctDNA sample (L-2) detected a single mutation: $A P C \mathrm{R} 216^{*}$. In a comparison of the genetic profiles of $\mathrm{P}-2, \mathrm{O}-2$, and $\mathrm{L}-2$, it was found that $A P C \mathrm{R} 216^{*}$ was present in all $\mathrm{P}-2, \mathrm{O}-2$, and $\mathrm{L}-2$, whereas it was not detected in L-2. Conversely $A P C$ R $216^{*}$ was detected in L-2, which is well-known genetic signature of colorectal cancer. These findings also support the usefulness of liquid biopsy-based gene mutation profiling in the preoperative diagnosis of metastatic colorectal cancer to the ovary. The patient received postoperative adjuvant chemotherapy (8 courses of capecitabine and oxaliplatin; XELOX).

\section{Discussion}

To the best of our knowledge, the current study is the first to characterize the genetic profile of metastatic colorectal cancer to the ovary, by subjecting liquid biopsy samples to a novel comprehensive gene mutation analysis technique; i.e., CAPP-Seq. We investigated the mutation profiles of tumor and liquid samples of metastatic colorectal cancer to the ovary to elucidate the potential of liquid biopsy examinations as a tool for preoperative diagnosis. In comparisons of the mutation profiles of the patients' tumor and liquid samples, matching colorectal cancer mutation signatures were observed in both patients, suggesting that liquid biopsy has potential as a tool for aiding the preoperative diagnosis and treatment of metastatic ovarian cancer from colorectal cancer.

Liquid biopsy examinations are non-invasive and have the potential to improve cancer (including recurrence) detection, non-invasive tumor genotyping, and disease monitoring. However, most early-stage and many advanced-stage solid tumors exhibit very low levels of ctDNA, which complicates ctDNA detection and analysis. In this study, we used CAPP-Seq, a novel approach which combines the hybrid affinity capture of hundreds of genomic regions with deep sequencing and a specialized bioinformatics workflow, for the ultrasensitive quantitation of ctDNA during comprehensive gene expression analysis (16). CAPP-Seq is able to 
detect ctDNA in patients with early and advanced stages of various human malignancies, including lung cancer and lymphoma $(17,20)$. However, in the gynecological field there have not been any reports about the comprehensive gene mutation analysis of liquid biopsy samples, which might be a powerful tool for advancing personalized medicine for gynecological malignancies.

We revealed that liquid biopsy examinations have potential to facilitate the preoperative diagnosis of metastatic ovarian cancer from colorectal cancer. After examining FFPE tumor samples, Crobach et al reported that the gene mutation profiles of primary and metastatic ovarian cancer differ (10). Inactivating $A P C$ mutations were found in $4.7 \%$ of primary endometrioid or mucinous ovarian tumors, whereas they were identified in $71 \%$ of colorectal cancer metastases; thus, $A P C$ mutation analysis can be used to differentiate between primary endometrioid/mucinous ovarian tumors and colorectal cancer metastases to the ovary. In patient 1, KRAS G13D, APC E1306*, and TP53 H193Y mutations were detected in both O-1 and L-1, with MET gene amplification in L-1, which has been reported to be a common gene mutation pattern in colorectal cancer. In patient 2, APC R216* was detected in all P-2, O-2, and $\mathrm{L}-2$, which is also well-known genetic signature of colorectal cancer. These findings suggest that it might be possible to predict the development of metastatic ovarian cancer from colorectal cancer based on the detection of $A P C$ gene mutations in liquid biopsy samples.

The detection of KRAS mutations in liquid biopsy samples also plays an important role in selecting the optimal treatment for metastatic ovarian cancer from colorectal cancer. Anti-EGFR antibodies combined with cytotoxic agents are one of the standard treatments for advanced colorectal cancer. Although the addition of anti-EGFR antibodies was found to be related to a significantly reduced risk of disease progression and improved overall survival in patients with KRAS wild-type tumors, this treatment had no benefit in patients whose tumors carried KRAS gene mutations in codons 12 and $13(21,22)$ Furthermore, less favorable clinical outcomes, particularly shorter survival, have been reported to be associated with the presence of KRAS mutations in codon 13 (23). Thus, KRAS mutation status was confirmed to be a powerful biomarker for predicting the efficacy of anti-EGFR antibody treatment (24). In patient 1 , a KRAS G13D mutation, which confers resistance to anti-EGFR antibodies and is associated with greater progression, was detected in the liquid biopsy sample.

Tumor heterogeneity might also hinder personalized molecular-targeted treatment, which depends on patients' somatic mutation profiles. In a previous study, we detected intra- and inter-tumor multi-clonality during mutational profiling of multi-regional colon cancer using next-generation sequencing of FFPE tumor samples (25). The KRAS-NRAS status of a tumor can vary between regions, making it difficult to decide whether anti-EGFR antibodies should be used. Examining tumor heterogeneity is challenging because we can not use whole surgically resected tumor samples for gene mutation analysis, and collecting tumor samples can be difficult in advanced cases involving repeated biopsies. In another previous study, by using liquid biopsy, we also detected newly appearing KRAS-NRAS mutation after first-line chemotherapy in metastatic colorectal cancer with wild-type of KRAS exon 2 in tumor tissue at the diagnosis, which could be a negative predictive marker for panitumumab (anti-EGFR antibody) (26). Non-invasive liquid biopsy examinations, whose results are not influenced by tumor heterogeneity, might be useful for determining the optimal chemotherapy regimen, including for novel molecular-targeted agents, immediately just before the treatment.

In conclusion, this is the first study to examine the tumor gene mutation profile of metastatic colorectal cancer to the ovary using both tumor and liquid samples. The difficulty of obtaining a preoperative diagnosis and tumor heterogeneity can both influence the effectiveness of molecular-targeted agents, such as anti-EGFR antibodies. Thus, in the clinical setting liquid biopsy examinations, which are non-invasive and easy to perform repeatedly, might be useful. The characterization of the genetic profiles of tumors based on liquid biopsy examinations might lead to the development of novel personalized treatment strategies. To confirm the findings of this study, a further investigation should be conducted.

\section{Acknowledgements}

Not applicable.

\section{Funding}

The present study was supported by Ministry of Education, Culture, Sports, Science and Technology of Japan Grants-in-Aid for Scientific Research Grant (grant no. JP18K16776).

\section{Availability of data and materials}

All data generated or analyzed during the present study are included in this published article.

\section{Authors' contributions}

NI, KS, KN and KI designed the research. NI, KS, TN, TY and ST conducted the experiments. NI and KS analyzed the data. The manuscript was drafted by KS, KN and KI. All authors read and approved the final manuscript.

\section{Ethics approval and consent to participate}

This study was approved by the ethics committee of Wakayama Medical University Faculty of Medicine (authorization number: 2025) and Kindai University Faculty of Medicine (authorization number: 29-066). All of the patients in this study provided written informed consent for the use of their plasma and tissue samples.

\section{Patient consent for publication}

All participants provided written informed consent for publication.

\section{Competing interests}

The authors declare that they have no competing interests. 


\section{References}

1. Kubeček O, Laco J, Špaček J, Petera J, Kopecký J, Kubečková A and Filip S: The pathogenesis, diagnosis, and management of metastatic tumors to the ovary: A comprehensive review. Clin Exp Metastasis 34: 295-307, 2017.

2. Hanna NN and Cohen AM: Ovarian neoplasms in patients with colorectal cancer: Understanding the role of prophylactic oophorectomy. Clin Colorectal Cancer 3: 215-222, 2004.

3. Tan KL, Tan WS, Lim JF and Eu KW: Krukenberg tumors of colorectal origin: A dismal outcome-experience of a tertiary center. Int J Colorectal Dis 25: 233-238, 2010.

4. Fujiwara A, Noura S, Ohue M, Shingai T, Yamada T, Miyashiro I, Ohigashi H, Yano M, Ishikawa O, Kamiura S and Tomita Y: Significance of the resection of ovarian metastasis from colorectal cancers. J Surg Oncol 102: 582-587, 2010.

5. McCormick CC, Giuntoli RL II, Gardner GJ, Schulick RD, Judson K, Ronnett BM, Vang R and Bristow RE: The role of cytoreductive surgery for colon cancer metastatic to the ovary. Gynecol Oncol 105: 791-795, 2007.

6. Rayson D, Bouttell E, Whiston F and Stitt L: Outcome after ovarian/adnexal metastectomy in metastatic colorectal carcinoma. J Surg Oncol 75: 186-192, 2000.

7. Ojo J, De Silva S, Han E, Lin P, Wakabayashi M, Nelson R and Lai LL: Krukenberg tumors from colorectal cancer: presentation, treatment and outcomes. Am Surg 77: 1381-1385, 2011.

8. Willmott F, Allouni KA and Rockall A: Radiological manifestations of metastasis to the ovary. J Clin Pathol 65: 585-590, 2012.

9. Ganesh K, Shah RH, Vakiani E, Nash GM, Skottowe HP Yaeger R, Cercek A, Lincoln A, Tran C, Segal NH, et al: Clinical and genetic determinants of ovarian metastases from colorectal cancer. Cancer 123: 1134-1143, 2017.

10. Crobach S, Ruano D, van Eijk R, Fleuren GJ, Minderhout I, Snowdowne R, Tops C, van Wezel $\mathrm{T}$ and Morreau $\mathrm{H}$ : Target-enriched next-generation sequencing reveals differences between primary and secondary ovarian tumors in formalin-fixed, paraffin-embedded tissue. J Mol Diagn 17: 193-200, 2015.

11. Misale S, Yaeger R, Hobor S, Scala E, Janakiraman M, Liska D, Valtorta E, Schiavo R, Buscarino M, Siravegna G, et al: Emergence of KRAS mutations and acquired resistance to anti-EGFR therapy in colorectal cancer. Nature 486: 532-536, 2012.

12. Murtaza M, Dawson SJ, Tsui DW, Gale D, Forshew T, Piskorz AM, Parkinson C, Chin SF, Kingsbury Z, Wong AS, et al: DNon-invasive analysis of acquired resistance to cancer therapy by sequencing of plasma DNA. Nature 497: 108-112, 2013.

13. Peeters M, Oliner KS, Parker A, Siena S, Van Cutsem E, Huang J, Humblet Y, Van Laethem JL, André T, Wiezorek J, et al: Massively parallel tumor multigene sequencing to evaluate response to panitumumab in a randomized phase III study of metastatic colorectal cancer. Clin Cancer Res 19: 1902-1912, 2013.

14. Qiu M, Wang J, Xu Y, Ding X, Li M, Jiang F, Xu L and Yin R: Circulating tumor DNA is effective for the detection of EGFR mutation in non-small cell lung cancer: A meta-analysis. Cancer Epidemiol Biomarkers Prev 24: 206-212, 2015.

15. Oxnard GR, Thress KS, Alden RS, Lawrance R, Paweletz CP, Cantarini M, Yang JC, Barrett JC and Jänne PA: Association between plasma genotyping and outcomes of treatment with osimertinib (AZD9291) in advanced non-small-cell lung cancer. $\mathrm{J}$ Clin Oncol 34: 3375-3382, 2016.
16. Newman AM, Bratman SV, To J, Wynne JF, Eclov NC, Modlin LA, Liu CL, Neal JW, Wakelee HA, Merritt RE, et al: An ultrasensitive method for quantitating circulating tumor DNA with broad patient coverage. Nat Med 20: 548-554, 2014.

17. Newman AM, Lovejoy AF, Klass DM, Kurtz DM, Chabon JJ, Scherer F, Stehr H, Liu CL, Bratman SV, Say C, et al: Integrated digital error suppression for improved detection of circulating tumor DNA. Nat Biotechnol 34: 547-555, 2016.

18. Xu C, Nezami Ranjbar MR, Wu Z, DiCarlo J and Wang Y: Detecting very low allele fraction variants using targeted DNA sequencing and a novel molecular barcode-aware variant caller. BMC Genomics 18: 5, 2017.

19. Narahara M, Higasa K, Nakamura S, Tabara Y, Kawaguchi T, Ishii M, Matsubara K, Matsuda F and Yamada R: Large-scale East-Asian eQTL mapping reveals novel candidate genes for LD mapping and the genomic landscape of transcriptional effects of sequence variants. PLoS One 9: e100924, 2014.

20. Scherer F, Kurtz DM, Newman AM, Stehr H, Craig AF, Esfahani MS, Lovejoy AF, Chabon JJ, Klass DM, Liu CL, et al: Distinct biological subtypes and patterns of genome evolution in lymphoma revealed by circulating tumor DNA. Sci Transl Med 8: 364ra155, 2016.

21. Karapetis CS, Khambata-Ford S, Jonker DJ, O'Callaghan CJ, Tu D, Tebbutt NC, Simes RJ, Chalchal H, Shapiro JD, Robitaille $\mathrm{S}$, et al: K-ras mutations and benefit from cetuximab in advanced colorectal cancer. N Engl J Med 359: 1757-1765, 2008.

22. Bokemeyer C, Bondarenko I, Makhson A, Hartmann JT, Aparicio J, de Braud F, Donea S, Ludwig H, Schuch G, Stroh C, et al: Fluorouracil, leucovorin, and oxaliplatin with and without cetuximab in the first-line treatment of metastatic colorectal cancer. J Clin Oncol 27: 663-671, 2009.

23. Bazan V, Agnese V, Corsale S, Calò V, Valerio MR, Latteri MA, Vieni S, Grassi N, Cicero G, Dardanoni G, et al: Specific TP53 and/or Ki-ras mutations as independent predictors of clinical outcome in sporadic colorectal adenocarcinomas: Results of a 5-year Gruppo Oncologico dell'Italia Meridionale (GOIM) prospective study. Ann Oncol 16 (Suppl 4): iv50-iv55, 2005.

24. Van Cutsem E, Köhne CH, Láng I, Folprecht G, Nowacki MP, Cascinu S, Shchepotin I, Maurel J, Cunningham D, Tejpar S, et al: Cetuximab plus irinotecan, fluorouracil, and leucovorin as first-line treatment for metastatic colorectal cancer: Updated analysis of overall survival according to tumor KRAS and BRAF mutation status. J Clin Oncol 29: 2011-2019, 2011.

25. Kogita A, Yoshioka Y, Sakai K, Togashi Y, Sogabe S, Nakai T, Okuno K and Nishio K: Inter- and intra-tumor profiling of multi-regional colon cancer and metastasis. Biochem Biophys Res Commun 458: 52-56, 2015.

26. Shitara K, Yonesaka K, Denda T, Yamazaki K, Moriwaki T, Tsuda M, Takano T, Okuda H, Nishina T, Sakai K, et al: Randomized study of FOLFIRI plus either panitumumab or bevacizumab for wild-type KRAS colorectal cancer-WJOG 6210G. Cancer Sci 107: 1843-1850, 2016.

This work is licensed under a Creative Commons Attribution-NonCommercial-NoDerivatives 4.0 International (CC BY-NC-ND 4.0) License. 